\title{
Mechanical Remote Laboratory Development using iLab Shared Architecture
}

\author{
http://dx.doi.org/10.3991/ijoe.v11i5.4808 \\ Brahim Nasri, Ridha Ennetta, Sofienne Bouallègue \\ Higher Institute of Industrial System of Gabes, Gabes, Tunisia
}

\begin{abstract}
This paper focuses on the implementation of the first remote laboratory in the mechanical field being used by the Higher Institute of Industrial Systems of Gabès (ISSIG)-Gabès University, Tunisia. The developed remote laboratory was achieved through the e-Sience Tempus project. The control system for this remote laboratory was implemented using LabVIEW software. The experiment studied a forced vibrations system. The students access the platform via the internet to determine the resonance frequency of damping forced vibration from the amplitude vs. excitation frequency and the phase angle vs. excitation curves. The students also determined the damping ration for the system via the phase angle vs. excitation frequency curve. All online experiments were deployed on an interactive version of the iLab Shared Architecture (ISA).
\end{abstract}

Index Terms-Remote laboratory, mechanical vibration, iLab Shared Architecture, e-science Tempus Project, LabVIEW.

\section{INTRODUCTION}

Remote laboratories use real equipment that can be operated remotely through an experiment interface [1]. A remote laboratory simplifies the logistics and requirements involved in conventional laboratory work, including equipment, lab space, staffing, training, and safety. The students can conduct their experiment from any computer on their own schedule instead of in a specialized laboratory on the staff's schedule. Remote labs can also enrich science and engineering education by vastly increasing the scope of experiments that students have access to throughout their academic careers. Moreover, an online laboratory facilities efficient sharing of expensive equipment. The core of the remote laboratory is a cluster of general-purpose and/or specialized instruments interfaced to a set of personal computer systems connected to the internet [2]. Experiments allow the students to compare reality with simulations, collaborate with each other, and follow their curiosity. Conducting experiments motivates the students and helps them to learn more effectively [3].

In this paper, we focus on the online laboratory being used by the ISSIG University in Tunisia. This laboratory, which is called Online-Lab, is a part of the e-Sience project (Réseau Maghrébin de Laboratories à Distance) carried out in cooperation with France, Austria, Tunisia, Algeria, Morocco, Romania and Greece. It is financed by Tempus. The Tempus project seeks to create a set of remote laboratories in Tunisia, Morocco and Algeria in cooperation with France, Austria, Romania and Greece. The project is financed by the European Union. It also seeks to help the Maghreb countries train a large number of students to satisfy the huge demand for training of senior technicians and engineers. It is the only online Lab in the mechanical field for the e-Sience project.

Online-Lab, the first remote laboratory in Tunisia that completely works at this moment, was implemented and developed to allow students to conduct practical work from anywhere and at any time without being physically present. Furthermore, due to the number of students and space required for the mechanical engineering equipment, the remote lab was the solution adapted by the ISSIG. Starting in April 2015, the ISSIG platform is available at http://onlienlab.issig.rnu.tn/iLabSer viceBroker/.

Different architectures can be used to support eearning environments [4]. Some of them are based on proprietary software solutions such as LabVIEW, while others are supported by open-source software such as PHP, JavaScript, Java, Phyton, and so on. National Instrument's LabVIEW software, which is professional software for analysis, data acquisition, real-time control and remote laboratories, has an enormous impact on engineering education. Benefiting from that, the ISSIG's Online-Lab with the cooperation of the STI Company has developed an innovative and flexible remote laboratory to record the amplitude vs. excitation frequency and a phase angle vs. excitation frequency curve to determine the resonance frequency for a forced vibrations module in a real-time framework via internet. The remote experiment can also estimate the damping ration from the angle phase vs. excitation frequency curve.

There are many software applications used for sharing online laboratories [5-6]. In our case, we have chosen the iLab Shared Architecture (ISA) of the Massachusetts Institute of Technology (MIT) that provides a framework for development and deployment [6]. The ISA software divides an online lab into three distinct parts: the Lab Client, the Service Broker, and the Lab-Server that simplify the development of remote labs around the word by providing reusable components for common lab administration function. The ISA tool provides a framework for deployment of iLabs in a disturbed fashion using web services. This allows the lab to be made available to users' worldwide using standard network protocol. In addition, the ISA component can also make the remote lab easier to share across institutions [6].

The remainder of this paper is organized as follows: Section II describes the architecture of the developed ISSIG's remote laboratory. Section III is dedicated to the presentation of the vibration module installed in the 
laboratory. In Section IV, the designed LabVIEW interface for remote control is described. Section V presents the pedagogical evaluation of the developed ISA-based mechanical remote laboratory.

\section{DISCRIPTION}

\section{A. iLab Shared Architecture (ISA)}

The ISA tool is a robust, scalable and open source architecture built on web services that provide a unifying software framework to support access to a wide variety of online laboratories. The ISA provides a set of generic lab services such as user account management, scheduling, and data storage in a middleware system that can be accessed using web services. There are three different types of architecture available in the framework of the online laboratories: batched, sensor, and interactive experiments.

In the first architecture, the batched iLabs are labs in which experiments are completely specified prior to submission and run without intervention. Batched iLabs are deployed with Lab Clients, Service Brokers, and Lab Servers that communicate over the Internet using web services. In this model, as shown in Figure 1, Lab Clients and Lab Servers communicate with each other exclusively through the iLab Service Broker [7].

In the second architecture, called sensor experiment, the students cannot specify or configure any of the parameters. However, they can monitor and control realtime data streams without influencing the phenomena that is being measured [8].

In the last architecture, as depicted in Figure 2, in addition to the Lab Client, Service Broker, and Lab Server, stand alone web services are added to manage experiment storage and lab scheduling. The student must first schedule the time to use the lab. At the schedule time, the student $\operatorname{logs}$ in and is able to launch a Lab Client. He interacts directly with the Lab Server, and once a lab session begins, the Service Broker steps out of the picture.

In our platform, the student can vary the exciter speed and record the variation of the amplitude of the beam response. For each speed, he can record the tachometer frequency. To accommodate this, we adopted the interactive experiments architecture that consists of three parts: the Interactive Lab Client Server, the Interactive Service Broker, and the Interactive Lab Server.

The Interactive Lab Client server is the interface through which students access the iLab. It provides an intuitive representation of the iLab that is being run, allowing users to specify parameters and interact with the lab hardware. The ISA supports multiple clients' technology, including Java Apples, Java Server Pages, Windows Form Clients, and LabVIEW Front Panels [9]. The Interactive Lab Server is the component that is connected directly to the lab equipment and deals with the actual operation of the experiment hardware. It is the administrative interface of the lab equipment and enables laboratory administrators to set up and configure each experiment independently. The Interactive Service Broker is the heart of the ISA. It provides generic administrative services, such as authentication, authorization, user management, and scheduling and data storage. The
Service Broker serves as the gateway in inter-institutional relationship. To support these functions for interactive

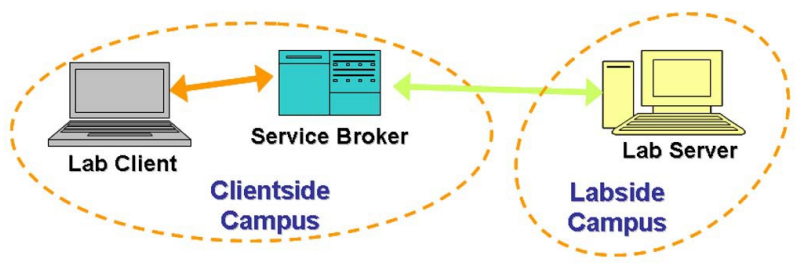

Figure 1. Topology of a batched experiment based on the iLab shared architecture.

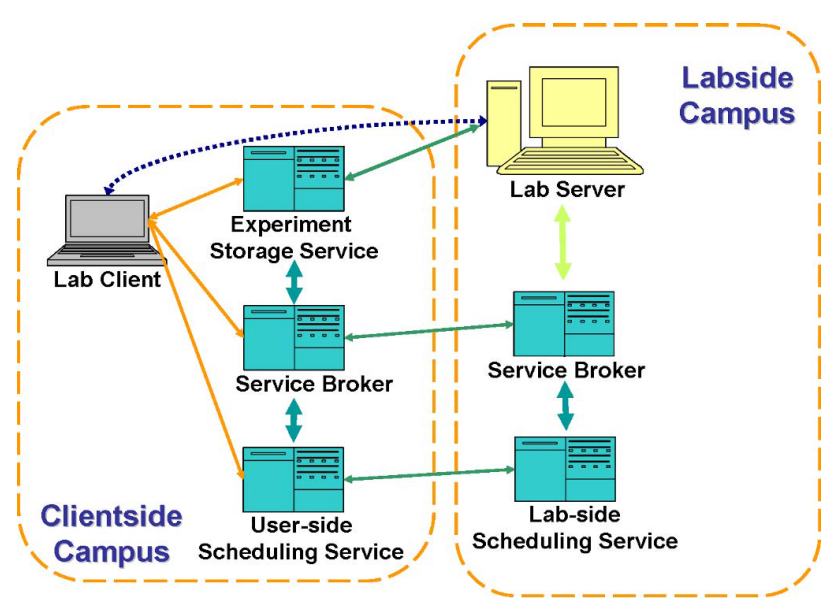

Figure 2. Topology of an interactive batched experiment based on the iLab shared architecture.

labs, three tiers have been added to the ISA: the Experiment Storage Service (ESS), User-side Scheduling Service (USS), and Lab-side Scheduling Service (LSS).

The ESS component is a stand-alone web service that allows Service Brokers, Lab Servers, and Lab Clients to store experiment data. It also provides storage of binary data (images, video or audio) and XML based text/numeric data. In addition, the students can execute interactive experiments by running the interactive lab client. To support interactive experiments that require scheduling access, the iLab interactive architecture envisions scheduling servers and services that enable students, from different campuses, to reserve time periods to execute experiments. Since the user side and lab side require different scheduling functionalities, the USS and LSS components are introduced to the ISA architecture to manage reservation [5-6].

The USS tool is used in conjunction with the LSS to allocate lab time to the users. Using the LSS, a student who wants to schedule time in a given lab must select from a set of available blocks of time. Additionally, the USS is responsible for notifying students if the reservation must be canceled and for considering course/lab requirements when distributing time blocks [5-6]. The LSS tool is responsible for defining the scheduling policy for a particular lab. It is designed to run in conjunction with multiple USS software and may schedule multiple lab servers. The LSS defines the broad lab availability for individual USS/Service Brokers. In turn, a given USS/Service Broker will distribute experiment time to students based on lab requirements, instrument availability, and instructor policy [5-6]. 


\section{B. ISSIG remote lab}

As described previously, our remote lab is based on the Interactive Shared Architecture to deploy and share the labs (Figure 3).

As shown in Figure 4, the platform is available on: http://onlinelab.issig.rnu.tn/iLabServiceBroker/. The students browse from any place where the Internet connection exists to register and request membership to the group associated with the laboratory. They can access the Service Broker and login page and supply their user names and passwords. When the students have permission to access to the booking service, they can choose the available experiment to launch it.

\section{DESCRIPTION OF THE VIBRATION FRAME MODULE}

The vibration frame module consists of a number of rigid vertical and horizontal member's each having profiled grooves running along their length. Within these grooves are fitted special profiled nuts that allow all of the component parts of the module to be fitted into position. As shown in Figure 5, the core components of the vibration module are described in the following.

\section{A. Rigid Beam}

The rigid steel beam has as dimensions $25.4 \times 12 \times 840 \mathrm{~mm}^{3}$. Its weight is about $2.1 \mathrm{Kg}$. The beam fits into a beam pivot arrangement that is attached to the inner vertical member of the frame. The beam pivot restricts the movement of the beam to one-degree-offreedom in the vertical plan of the vibration frame.

\section{B. Spring}

As the free end of the rigid steel beam is suspended as spring, the spring is attached to the beam using a special clamp. The clamp can be adjusted so that the spring can be moved along the beam and fixed into position. The spring dimensions are as follows: $31.75 \mathrm{~mm}$ outside diameter, $3.25 \mathrm{~mm}$ wire diameter and $152.4 \mathrm{~mm}$ free length.

\section{Damper}

The damper comprises a rigid stem that is attached to the rigid steel beam via the stem holder. The location along the beam can be adjusted using the simple clamping mechanism of the stem holder.

\section{Motor Exciter}

The motor exciter is rigidly attached to the beam and can be adjusted to be positioned anywhere along the beams main length. The motor exciter consists of a horizontal motor onto which is attached an 18-tooth pulley wheel. The component suspended below the motor is a bearing block which contains a ground steel shaft and two precision bearings. Attached onto the shaft at either side of the bearing block are two to 72 -tooth aluminum pulley wheels. A pulley belt runs between the 18-tooth and 72tooth pulley wheels. This gives a gear ratio of 1:4. For every revolution of the motor, the eccentric mass revolves 0.25 turns.

Attached to each of the 72-tooth pulley wheels are two eccentric masses. The eccentricity produced comes from the fact that a hole has been introduced into each mass. As the eccentric masses rotate, they create a force on the beam that increases with rotation frequency. The force is directed downward onto the beam when the hole is dead centre at top. The rotation speed of the eccentric masses is

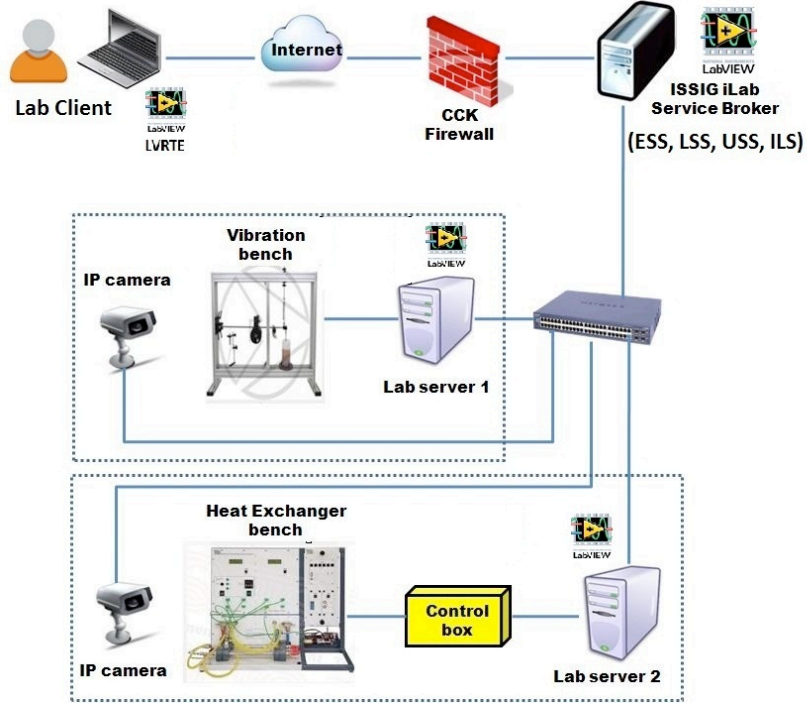

Figure 3. ISSIG remote laboratory architecture.

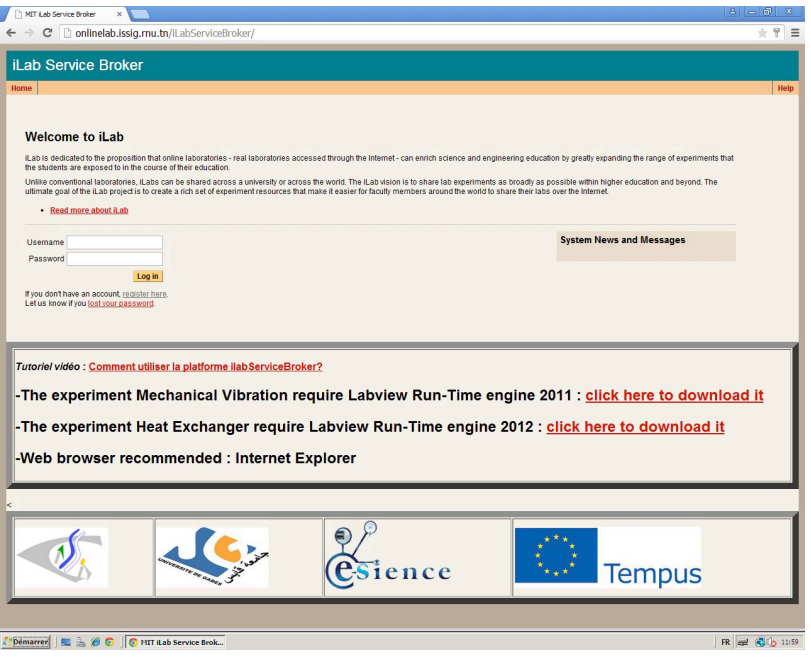

Figure 4. Online iLab ISSIG plate-form.

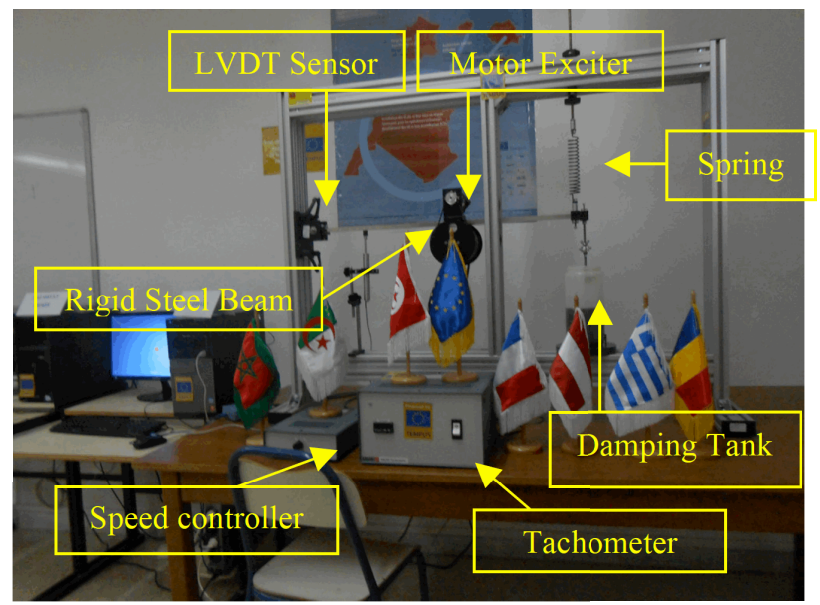

Figure 5. Vibration frame module.

controlled by the speed controller unit, which itself is powered from the tachometer unit.

The tachometer then supplies $24 \mathrm{~V}$ DC to the speed controller, which in turn supplies $24 \mathrm{~V} \mathrm{DC}$ to the motor. The frequency of rotation of the eccentric masses is observed from the digital speed display. It is displayed in 
units of Hz. A proximity sensor attached to the back of the motor exciter is used to generate the signal for the tachometer. As the hole in the rear eccentric mass passes the proximity sensor, a signal pulse is generated and returns to the tachometer. The signal pulse is the shape of a square wave. The overall mass of the motor exciter including cables is $5.105 \mathrm{Kg}$.

\section{E. Tachometer}

The tachometer unit displays the excitation frequency of the input force to the beam and powers the speed controller. It has an output for the proximity sensor. When the tachometer unit is powered up, the speed controller unit can also run

\section{F. Speed Controller}

The speed controller is powered from the tachometer when it is connected to this unit. It has a rotary potentiometer in its front panel which, when turned, increases the speed of the motor exciter.

\section{G. LVDT sensor}

For monitoring the vertical beam displacement, an LVDT (Linear Variable Displacement Transducer) sensor is mounted from the lower horizontal member of the vibration frame. As the beam moves vertically, the LVDT signal can be monitored by using oscilloscope software.

\section{H. Pisco-Scope displaying unit}

The Pico-Scope consists of a USB type of oscilloscope that enables different modes of acquisition, analysis, and save data options.

\section{LABVIEW REMOTE INTERFACE}

The vibration frame module was adapted for remote operation. The forced vibration module client interface was implemented using the LabVIEW tool from the National Instrument Company. Figures 8 and 9 show the implemented LabVIEW client interface of the forced vibration module. In the client side, students use the "motion control" area to vary the exciter speed slowly through its available range and by clicking to Auto Scale button (Pico-Scope) to record the beam response signal (red trace) and the proximity sensor (blue trace). At each speed, the tachometer frequency is also recorded in the LabVIEW interface client.

\section{A. LabVIEW Web Server}

Using the LabVIEW Web Server to publish VIs to be remotely controlled via the Internet, the client needs to install the LabVIEW runtime engine [10]. The Web Publishing Tool is a LabVIEW built-in tool to publish the front panel of a VI as a HTML document in the web. There are three steps from choosing a VI until saving the HTML file to disk. In the first step "Select VI and Viewing Options", the VI to publish, which must be in memory, has to be selected. Also the "Viewing Mode" can be changed between "Embedded", "Snapshot" and "Monitor", where Embedded allows clients to view and control the front panel, Snapshot to only display a static image of the front panel and Monitor to display a snapshot with a configurable updating interval. For the next step "Select HTML Output", we can type in a title (document title), a text before (header) the front panel and a text after (footer) the front panel that is going to be displayed on the

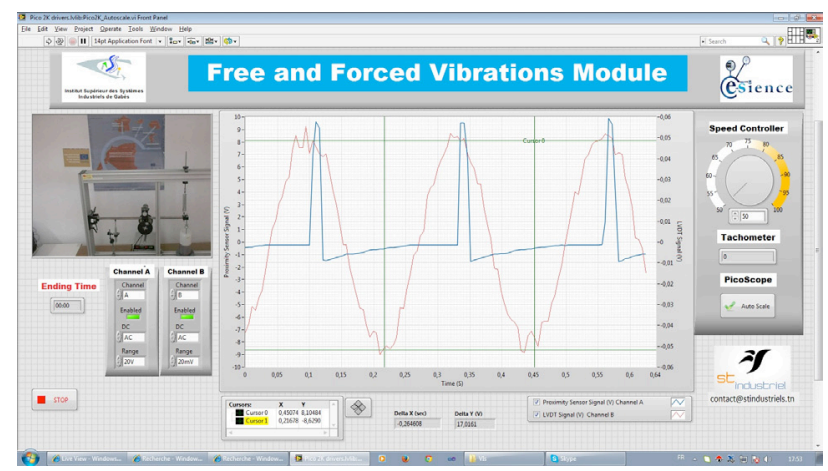

Figure 6. Forced vibration LabVIEW client interface.

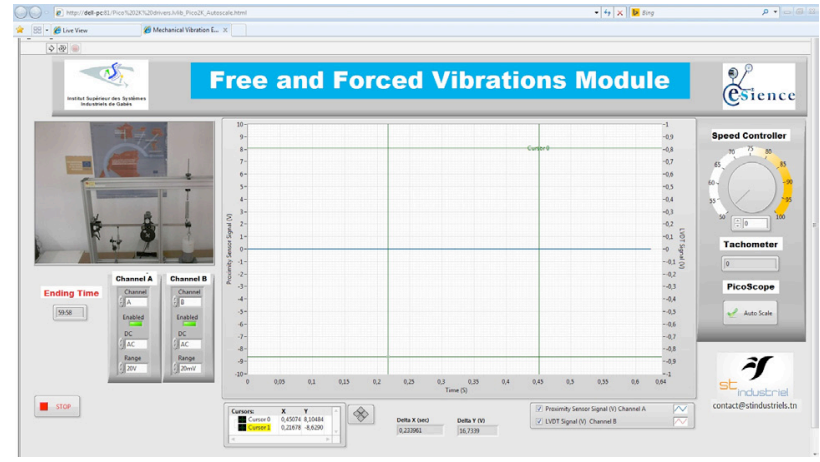

Figure 7. The virtual front panel interface loaded in a web-browser.

respective place. The next step is the "Save the New Web Page" where the created HTML file of the VI is going to be saved to a directory with the selected filename and a URL will be created [10].

After saving, the VI is now ready to be remotly controlled from a client by typing the URL into the address or URL field of his Web browser window. The URL for the front panel is as follows: $\mathrm{http}: / /$ dellpc:81/Pico $\% 202 \mathrm{~K} \% 20$ drivers.lvlib Pico2K Autoscale.ht ml. This URL must be integrated in LabApp table on the

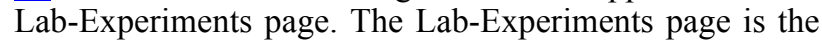
configuration page for experiment. To complete this page, we must indicate the title, the client guide, the application key and the path that contains the target application. To access the LabApp table, we must also configure the experiment on Manage Lab Clients in Service Broker part.

Four items must complete the same for the LabExperiments: The title of the experiment, the client guide, the version and the loader script. Here, we choose an interactive redirect client type experiment and put the same URL as the Web Page URL putted on the LabExperiments page.

\section{B. Running experiment}

The iLab forced vibration module is an interactive experiment that requires users to schedule experiment in advance. To run the experiment, the students $\log$ in to Service Broker, select the mechanical vibration experiment group and reedem the reservation they scheduling in advance. The Service Broker checks to make sure that the user has a valid reservation and that he/she is autorized to use the mechanical vibration experiment. The user is then presented with a "Lunch Lab" button and is able to start the experiment client (Figure 10). 


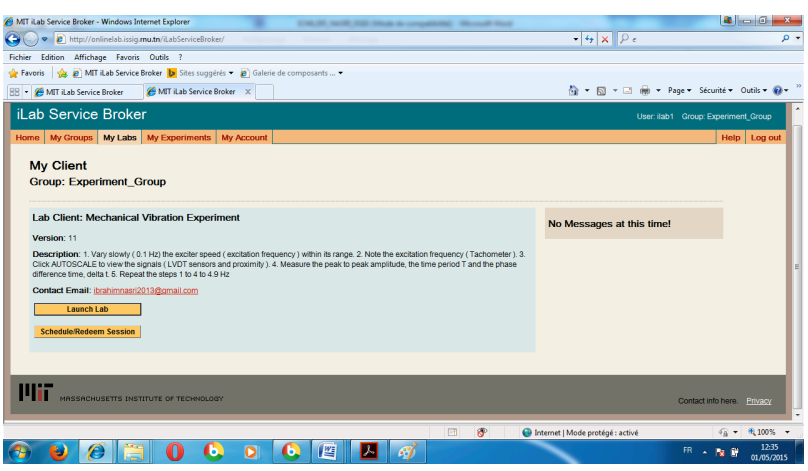

Figure 8. Student view of the mechanical vibration experiment page on the service broker.

When the experiment is lunched, the Service Broker facilities the exchange of credentials so that the Lab Client running in the students' browser can talk directly with the Lab Server and experiment hardware. The mechanical vibration experiment installation has a web camera so users can watch the experiment while in progress. We found that the camera is essentiel to student understanding and to beliving that it is working and controlling real hardware.

\section{Experimental procedure}

\section{1) Experiment 1}

The objective of this experiment is to determine the resonance from normalized amplitude vs. excitation frequency curve of the damping forced vibrations and compare it with the theoretical values. To do this experiment, we vary the exciter speed (Figure 11) slowly through its available range by using the needle and observing the variation of the amplitude of the beam response. By viewing the approximate frequency at which resonance occurs, it is possible to fine tune the range of frequencies over which it is useful to take results. After that, we can increase the speed range by suitable increments $(0.1 \mathrm{~Hz})$.

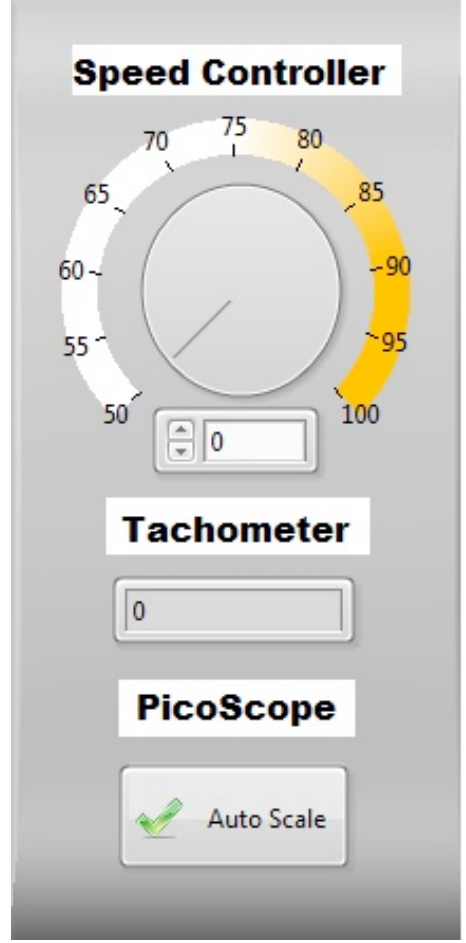

Figure 9. LabVIEW control for the client interface.
At each speed, we record the tachometer frequency $(\mathrm{Hz})$, and the peak-to-peak amplitude of the signal response. We recorded the peak-to-peak output amplitude; we clicked on "Auto Scale". It is the complete amplitude of the LVDT signal. An example of the signal is illustrated in Figure 12 about how to measure the peak-to-peak amplitude.

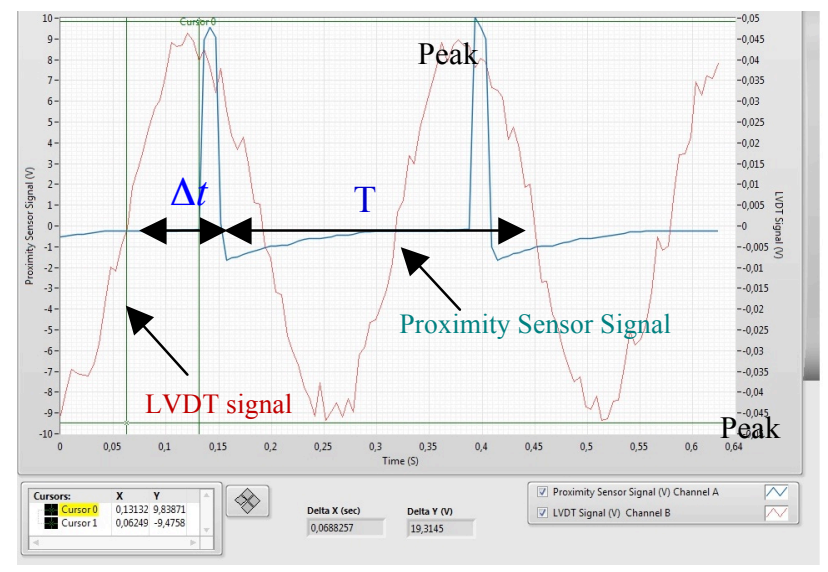

Figure 10. Measurement LabVIEW interface client.

The normalized amplitude is obtained by dividing the peak-to-peak response amplitudes by the square of the excitation frequencies because the inertia force applied to the beam by the exciter is proportional to the square of frequency. We can identify the resonance frequency from the response peak after plotting the normalized amplitude against the excitation frequency (Figure 13). The resonance frequency $\omega_{0}$ of damping forced vibration is equal to $4.1 \mathrm{~Hz}$.

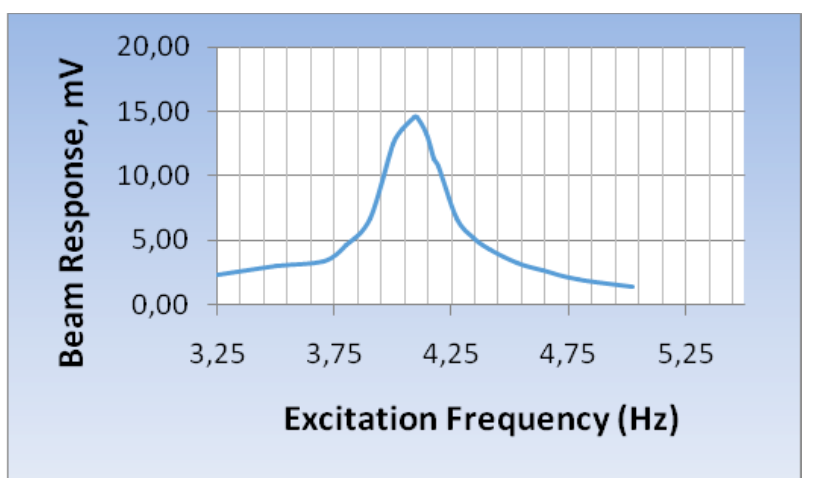

Figure 11. Beam displacement response (peak-to-peak) vs. excitation frequency.

\section{2) Experiment 2}

The objective of this experiment is to observe the phase angle of the system and obtaining the resonance frequency from the phase shift vs. the exciter frequency and determine the system damping ratio from the angle phase curve. To do this experiment, we followed the steps below:

a. Having viewed the system response from part of the experiment 1 , the student knows where about resonance occurs for the apparatus set up.

b. The student runs the motor exciter through the same excitation frequency used for experiment 1.

c. While doing this, the student monitors the position of a leading edge of the proximity sensor signal with the peak of the closet beam response 
(LVDT signal). He continues to monitor this pairing while going through resonance and out of resonance.

d. After it is brought back to the lowest excitation frequency, the student records the measurement in the form of Table 1, where the excitation frequency and the time difference, between the leading edge of the proximity sensor and the peak of the beam response, are given in columns 1 and 2, respectively. Figure 12 explains what is exactly the time difference between the leading edge of the proximity sensor and the peak of the beam response and the period time of excitation.

e. The student continues to record while increasing the excitation frequency through the range of frequencies used in the experiment 1. According to Equations 1 and 2, we can record all the results in Table 1.

TABLE I.

PHASE ANGLE OF THE EXCITER MOTOR.

\begin{tabular}{|c|c|c|c|c|}
\hline$\omega(\mathrm{Hz})$ & $\Delta t(s)$ & $\begin{array}{c}\mathbf{T} \\
(\mathrm{sec})\end{array}$ & $\begin{array}{c}\text { Phase } \\
(\mathrm{rad})\end{array}$ & $\begin{array}{c}\text { Phase } \\
(\mathrm{deg})\end{array}$ \\
\hline- & - & - & - & - \\
\hline
\end{tabular}

An example of what to look for when running this part of the experiment is shown in Figures 14 and 15.

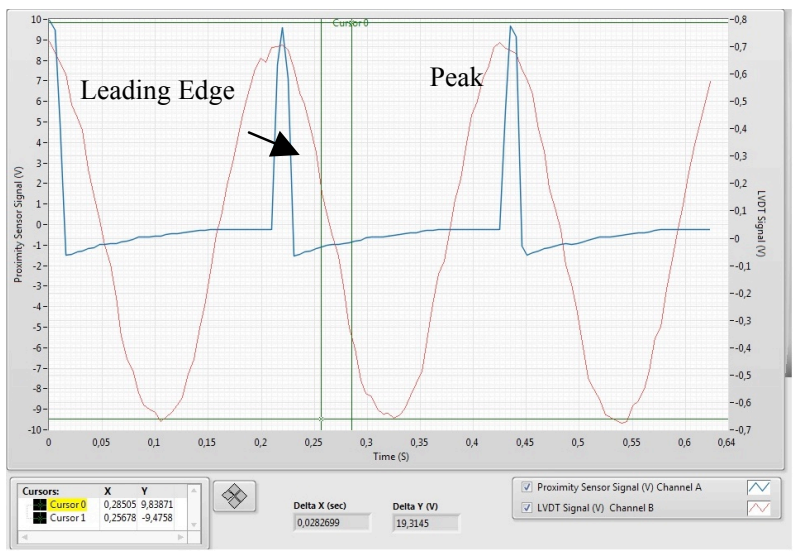

Figure 12. Leading edge and Peak very close to each other.

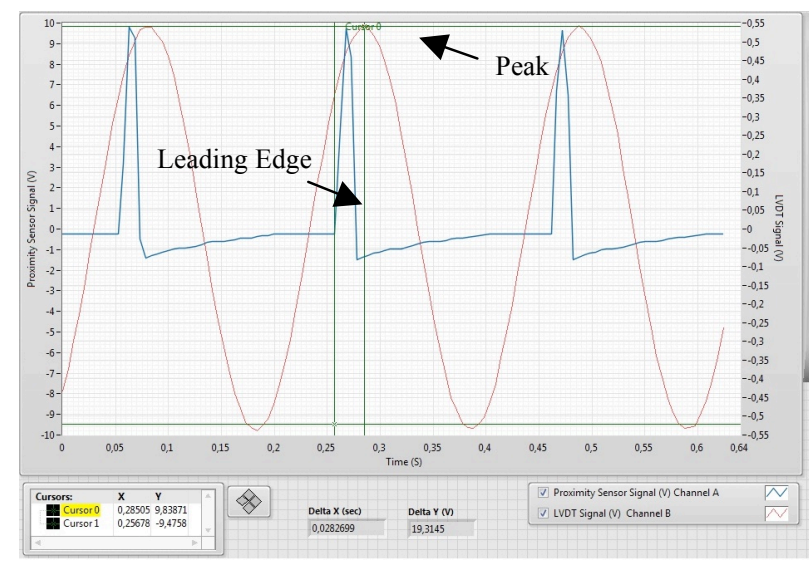

Figure 13. Leading edge and peak moving further apart.
From Figure 12, the phase expression is given by:

$$
\begin{gathered}
\phi=2 \pi \frac{\Delta t}{T}-\frac{\pi}{2} \quad[\mathrm{rad}] \\
\phi=360 \frac{\Delta t}{T}-90 \quad[\mathrm{deg}]
\end{gathered}
$$

where $\Delta t$ represents the time difference between proximity sensor and LVDT signals and $T$ the time period of the excitation.

When Table 1 is completed, the student can plot a graph of phase angle in degrees against excitation frequency and can obtain the resonance frequency, like in Figure 16. Here the student can compare the value of resonance frequency to the one obtained in the first experiment.

From Table 2, the 90 degrees phase corresponds to an excitation frequency of $4.07 \mathrm{~Hz}$. This compares with 4.1 $\mathrm{Hz}$ obtained by amplitude vs. excitation frequency curve. The results from this test can be also used to confirm the damping ratio $\zeta$ obtained in non-remote Lab test while using Equations (3) and (4):

$$
\begin{aligned}
& \zeta=\frac{1-\Omega^{2}}{2 \Omega} \tan \phi \\
& \Omega=\frac{\omega}{\omega_{0}}
\end{aligned}
$$

where $\omega$ and $\omega_{0}$ are the excitation and the resonance frequencies $(\mathrm{Hz})$, respectively.

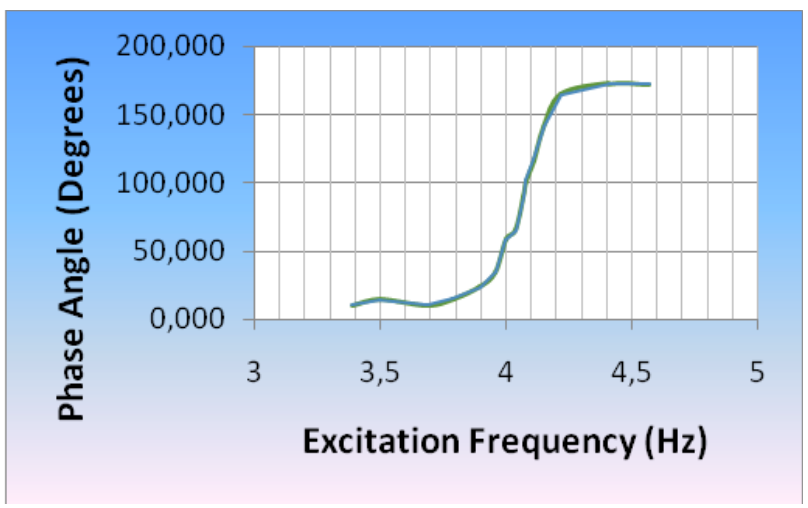

Figure 14. Phase angle against excitation frequency.

TABLE II.

DAMPING RATIO FROM PHASE CURVE.

\begin{tabular}{|c|c|c|c|c|c|}
\hline \begin{tabular}{c|c|c|c|}
$\omega$ \\
$(\mathrm{Hz})$
\end{tabular} & $\Omega$ & $\frac{\left(1-\Omega^{2}\right)}{2 \Omega}$ & $\phi$ & $\tan \phi$ & $\zeta$ \\
\cline { 1 - 2 } 3,96 & 0,973 & 0,026 & 0,602 & 0,687 & 0,018 \\
\hline 4,04 & 0,993 & 0,007 & 1,140 & 2,173 & 0,016 \\
\hline 4,08 & 1,002 & $-0,002$ & 1,775 & $\begin{array}{c}- \\
4,827\end{array}$ & 0,012 \\
\hline 4,11 & 1,010 & $-0,010$ & 2,035 & $\begin{array}{c}- \\
1,996\end{array}$ & 0,020 \\
\hline 4,15 & 1,020 & $-0,020$ & 2,456 & $\begin{array}{c}- \\
0,818\end{array}$ & 0,017 \\
\hline 4,22 & 1,037 & $-0,039$ & 2,868 & $\begin{array}{c}- \\
0,280\end{array}$ & 0,011 \\
\hline
\end{tabular}


The resonance frequency is taken from the first or second experiment. The student can calculate the damping ratio for each excitation frequency value, and when he/she finished to filling the table, he/she can deduce the average value for the damping ratio. The average value of the damping ratio from column 6 of Table 2 is 0.015 . This compares with 0.017 obtained by the experiment.

\section{Pedagogical evaluation}

The evaluation of our remote labs will be in the framework of the whole evaluation strategy adopted by all eSience project partners [11].

The evaluation of this remote lab has been performed in April 2015. The students were divided in two groups: the experimental group and the control group to participate in the large scale evaluation. The experimental group contained 18 students who used the remote lab in their studies, whereas the control group contained 18 students who did not used the remote lab in their studies. They followed a traditional learning method in the same course as the experimental group. The experimental group completed the online questionnaires. These questionnaires focus on five different but interrelated directions (a) e-sience content; (b) e-sience-Learner's Attitude Questionnaire; (c) esience-Knowledge test adapted in all courses; (d) esience- USE Questionnaire. The results of the evaluation are under study.

\section{CONCLUSION}

The main objective of this e-Sience Tempus project was to create an efficient remote lab network in the Maghreb region. As a partner in this project, ISSIG has to adapt and develop distant access solutions for the two remote labs, allowing students to perform practical experiments on mechanical vibration and heat transfer.

This work has presented the LabVIEW-based realization of a remote laboratory deployed and shared using an ISA tool. It is dedicated to students of the e-sience group to allow them to conduct and control a real-time forced vibrations module anywhere and anytime. The students accessed our platform to determine the resonance frequency of damping forced vibration and deduced the damping ratio for the system from the phase angle vs. excitation frequency curve. The system was implemented and controlled by LabVIEW virtual interface to run the entire platform. To test and validate this remote lab, a large scale valuation phase was conducted and the results are being proccessed.

\section{REFERENCES}

[1] P. Arpaia, A. Bacccigalupi,F. Cenneamo, P.A, Daponte, and "Measurement laboratory on geographic Network for remote test experiments," IEEE transactions on instrumentation and measurement, vol. 49, no. 5, pp. 992-997, 2000. http://dx.doi.org/10.1109/19.872919

[2] S.H. Chen, R. Chen, V. Ramakrishnan, S.Y. Hu, Y. Zhuang, C.C Ko, and Ben M. Chen, "Development of Remote Laboratory Experimentation through Internet", C-IEEE-hksrc., 1999.

[3] K. DeLong, V. Judson Harward, P. Bailey, J. Hardison, G. Kohse and Y. Ostrocsky, "Three Online Neutron Beam Experiments Based on the iLab shared Architecture", IEEE EDUCON Education Engineering, 2010. http://dx.doi.org/10.1109/educon. 2010.5492587

[4] B.R Chandre, K.P. Geevarghese, and K. V. Gangadhara, "Design and implementation of Remote Mechatronics Laboratory for eLearnig using LabVIEW and Smartphone and Cross-Platform Communication Toolkit", SCCT-2014, 2014.

[5] D. Cazacu, "A remote laboratory for frequency-response analysis of vibrating mechanical system", The $7^{\text {th }}$ International Conference Interdisciplinarity in Engineering, INTER-ENG'2013, 2013.

[6] R. Shroff, H. Soumare, and J. Hardison, J. del Alamo, and J. Harward, "A Versatile Internet-Accessible Electronics Workbench with Troubleshooting Capabilities." REV 2009, Bridgeport, CT, June 2009.

[7] J. L. Hardison, K. DeLong, P. H. Bailey, and V. Harward, "Deploying Interactive Remote Labs Using the iLab Shared Architecture", The $38^{\text {th }}$ SEE/IEEE Frontiers in Education Conference. http://dx.doi.org/10.1109/fie.2008.4720536

[8] T. Mao, "Scheduling Services and security Ticket Token services in iLab Interactive service", Master of Science in civil and Environmental Engineering, 2007.

[9] A. Naddami, A.Falhi, M. Gourmai, and M. Moussetad, "Determination of Synchronous Machine Parameters using iLab Shared Architecture". http://dx.doi.org/10.3991/ijoe.v10i3.3400

[10] National Instruments, www.ni.com, April 2015.

[11] T. Tsiatsos, S. Douka, A. Mavridis, S. Tegos, A. Naddami, T. Zimmer and D. Geoffroy, "Evaluation plan and preliminary evaluation of a network of remote labs in the Maghrebian countries", in press.

\section{AUTHORS}

Brahim Nasri, Ridha Ennetta, and Sofienne Bouallègue are with the Higher Institute of Industrial System of Gabes, Gabes, Tunisia.

This work was supported in part by e-sience (rESeau maghrébIn de laboratoirEs à distance) European Project under Tempus IV Program. Project No: 530341-TEMPUS-1-2012-1-FR-TEMPUS-JPCR. Submitted 17 June 2015. Published as resubmitted by the authors 20 August 2015. 